Cahiers $d u$ MONDE RUSSE

\section{Cahiers du monde russe}

Russie - Empire russe - Union soviétique et États indépendants

$52 / 4 \mid 2011$

Varia

\title{
Sandra Dahlke, Individuum und Herrschaft im Stalinismus
}

\author{
Gábor T. Rittersporn
}

\section{(2)enEdition}

Journals

Édition électronique

URL : http://journals.openedition.org/monderusse/7625

DOI : $10.4000 /$ monderusse. 7625

ISSN : $1777-5388$

\section{Éditeur}

Éditions de l'EHESS

\section{Édition imprimée}

Date de publication : 20 décembre 2011

Pagination : 750-755

ISBN : 978-2-7132-2353-2

ISSN : $1252-6576$

Référence électronique

Gábor T. Rittersporn, "Sandra Dahlke, Individuum und Herrschaft im Stalinismus », Cahiers du monde russe [En ligne], 52/4 | 2011, mis en ligne le 30 novembre 2012, Consulté le 24 septembre 2020. URL http://journals.openedition.org/monderusse/7625; DOI : https://doi.org/10.4000/monderusse.7625

Ce document a été généré automatiquement le 24 septembre 2020

(c) École des hautes études en sciences sociales 


\title{
Sandra Dahlke, Individuum und Herrschaft im Stalinismus
}

\author{
Gábor T. Rittersporn
}

\section{RÉFÉRENCE}

Sandra DAHLKE, Individuum und Herrschaft im Stalinismus. Emel'jan Jaroslavskij

(1878-1943). Munich : Oldenbourg, 2010, 484 p. (Ordnungssysteme. Studien zur Ideengeschichte der Neuzeit, vol. 29)

1 Pourquoi s'intéresser à la vie d'un bolchevik peu sympathique, en particulier si sa carrière révolutionnaire ne fut pas émaillée d'aventures excitantes, s'il est peu sorti de son bureau sous le nouveau régime, s'il n'a tué personne, n'a pas été tué lui-même et si, de surcroît, il a de quoi décourager l'historien face aux 1766 publications, dont 140 livres plus ou moins volumineux, qu'il a écrites. Sandra Dahlke réussit à montrer qu'une telle vie peut réserver de précieux enseignements.

2 Les auteurs aiment écrire des biographies car leur structure est donnée comme celle d'un formulaire. La plupart des mortels naissent, grandissent, apprennent quelque chose ou non, parcourent un itinéraire quelconque et disparaissent, tôt ou tard. Le chroniqueur n'a qu'à remplir les cases et tirer les quelques conclusions qui s'imposeront. Sandra Dahlke fait tout pour se compliquer la tâche. Mais, elle rend ainsi la vie d'un bolchevik ennuyeux infiniment plus lisible que les récits traditionnels.

Sandra Dahlke déconstruit le parcours de son personnage en blocs. Chacun consiste en expériences qu'il a faites, en problèmes qu'il a affrontés, en ce qu'il a compris ou n'a pas compris de son environnement et de sa propre vie intérieure et en questions que son existence pose à l'observateur. Dahlke ne néglige pas le contexte historique du quotidien de l'individu qui devient de plus en plus fascinant pour le lecteur. Mais contrairement aux biographies conventionnelles qui ont une tendance à s'égarer dans la description d'une époque au lieu de parler du héros, elle s'en tient rigoureusement à ce que celui-ci a subi et à l'influence qu'il a pu exercer sur les événements de son temps. 
Elle met en évidence des interactions complexes qui auraient échappé facilement à l'auteur d'une narration linéaire.

4 Né en 1878, un an avant Trockij et Stalin, et mort pendant la guerre, trois ans après le premier et dix ans avant le second, Emel'jan Jaroslavskij était un bolchevik illustre qui n'appartenait pas à l'état-major du régime. Les cinq mois qu'il avait passé comme secrétaire du Comité central avaient suffi au début des années 1920 à convaincre les sommités du parti qu'il ne pouvait servir la révolution en gérant les affaires de l'appareil. Jaroslavskij ne voyait pas nécessairement les choses du même œil. Il détestait la routine administrative et ne comprenait guère à quel point elle fusionnait avec le politique. L'apparatchik raté se concevait comme leader capable car sa spécialité était de mobiliser les masses. Mais il était dévoué à la cause et discipliné, si bien qu'il n'aspira jamais à une haute fonction.

5 Si Sandra Dahlke s'arrête sur les origines juives de Jaroslavskij, elle évite judicieusement de leur attribuer une importance décisive. Elle montre bien qu'à l'instar de la grosse majorité des radicaux russes, l'expérience formatrice de celui qui est né Mikhnej Gubel'man avait été la socialisation au sein de cercles d'étude révolutionnaires et le rassemblement autour de leurs maîtres à penser. Le milieu et la civilisation juifs l'ont marqué incomparablement moins que cet environnement et l'identification avec l'habitus de l'intelligentsia russe, son respect de la culture, sa volonté de servir les opprimés, son éthique de solidarité et sa détermination de se subordonner au collectif de ses pairs et à la cause commune. Jaroslavskij a fait un apprentissage d'autodidacte au sein de groupes radicaux, quand ceux-ci fonctionnaient parfois comme des communautés.

6 Au moment d'Octobre rouge, Jaroslavskij était déjà un ancien combattant éminent : membre du Parti social-démocrate dès sa fondation, bolchevik de la première heure, activiste clandestin ayant participé à des comités régionaux du mouvement, à l'organisation de cellules et de grèves, à la diffusion d'idées séditieuses et à la rédaction de publications de propagande. Le seul incident hasardeux de sa clandestinité fut son appartenance pendant deux ans à la direction d'une structure armée où ses activités sont peu connues mais lui ont coûté cinq années de bagne et le bannissement à vie.

7 En fait, quels que soient les postes qu'on lui a assignés au cours de la période mouvementée qui débute en 1917 et finit avec les débuts de la consolidation du nouveau régime vers 1923, il fut avant tout un agitateur. Ce fut, d'une manière ou d'une autre, sa principale fonction jusqu'à la fin de ses jours. Ses lettres et ses journaux intimes révèlent un orateur infatigable, passionné et extatique, enivré par sa propre ardeur, par le contact avec son public et par l'effet de ses paroles. Son énorme bibliographie témoigne de sa capacité à transformer son zèle de tribun en fièvre d'écriture. Le personnage exalté, attaché à son passé révolutionnaire, à ses titres de noblesse bolcheviks, à son rôle de propagandiste et à son sentiment d'être héritier de la tradition de l'intelligentsia sont autant de fils conducteurs des analyses de Sandra Dahlke.

8 Ils sont indispensables par exemple pour qu'elle s'y retrouve dans la complexité de l'être de son protagoniste où elle identifie les principaux éléments de l'image que Jaroslavskij a formée de sa personne et de sa persona et à laquelle il voulut correspondre tout au long de sa vie adulte. Le fort attachement du vieux bolchevik au collectif des anciens combattants et à leur association, dont il était en charge mais que Stalin va dissoudre en 1935, fut une source de fortes émotions. L'auteur a toutes les 
raisons de se demander si ces émotions et le pouvoir qu'elles exerçaient sur Jaroslavskij ne risquaient pas d'entrer en conflit avec le pouvoir politique. Cependant, d'autres composantes du moi militant furent étroitement liées à l'exercice de ce pouvoir. De sorte qu'il est justifié de poser la question de savoir si la participation de Jaroslavskij aux manœuvres politiques des années 1920 et 1930 n'équivaut pas à la trahison des traditions auxquelles il croyait s'identifier et à une métamorphose des passions révolutionnaires dont il fut incapable de se rendre compte.

Deux blocs importants des données réunies par Sandra Dahlke pour constituer la biographie du notable concernent ses activités pendant les conflits entre l'opposition de gauche et la fraction de Stalin et son concours à la production de l'idéologie du régime. Ces deux ensembles se recoupent en partie, du point de vue chronologique. Mais ils se distinguent par leur nature, par les milieux où Jaroslavskij se mouvait ainsi que par les positions qu'il occupait au sein de l'État-parti.

$10 \mathrm{Au}$ cours des luttes fractionnelles des années 1920, le vieux bolchevik agissait en politicien aux côtés de Stalin et de ses alliés. Il était de ceux qui faisaient la basse besogne : figurant parmi les dirigeants d'organismes qui contrôlaient et disciplinaient les militants, il persécutait ainsi les oppositionnels. En même temps, il se hisse au rang du théoricien éminent qui secondait le Secrétaire général pour échafauder la construction doctrinale du léninisme, un concept remarquablement flou mais suffisamment flexible pour le brandir afin de désavouer les contestataires qui tentaient de se justifier en invoquant les textes du maître à penser incontesté des bolcheviks. Le principal mérite de l'opération fut d'élever Stalin à la dignité de légataire et de seul successeur légitime du fondateur de l'État soviétique.

11 Symbole de la reconnaissance des services rendus par Jaroslavskij et de l'importance qu'il a gagnée, il a l'honneur d'être l'un des treize auteurs des articles de la Pravda qui fêtait le cinquantième anniversaire de Stalin, présentant ce dernier comme le Lenin des temps nouveaux. Autres symboles, il commence à assumer de nombreux postes suite aux conflits internes du parti en les cumulant avec ses fonctions au sein de l'appareil. Membre dirigeant de l'Académie communiste, enseignant à l'Institut des professeurs rouges, membre des rédactions de plusieurs revues littéraires, théoriques ou historiques prestigieuses et de la Pravda ainsi qu'un des historiens officiels du parti, Jaroslavskij est virtuellement partout où l'on parle d'autorité du passé, du présent et du futur du régime ainsi que du rôle qu'il devait jouer comme incarnation du progrès.

12 Sandra Dahlke est circonspecte lorsqu'elle écrit sur l'idéologie. À la différence de nombre de collègues, elle évite de la prendre comme motif principal ou unique des actes de ceux qui s'en réclament. Elle suit Clifford Geertz et saisit l'idéologie comme moyen de rationaliser les contraintes de situations pour lesquelles les traditions culturelles ne livrent pas de concepts. Du coup, elle réussit à séparer les avatars de l'idéologie bolchevique des modalités de son instrumentalisation. Jaroslavskij était de bonne foi et convaincu d'agir conformément aux idéaux qu'il professait en rédigeant ses innombrables articles et un manuel canonique du parti qui devaient confirmer la validité des prétentions de l'état-parti. Il était également de bonne foi quand il rectifiait ses concepts au gré des circonstances et quand il entrait en discussions houleuses avec d'autres combattants de ce qu'on nommait « le front idéologique ».

13 Il n'en reste pas moins que sa correspondance et ses écrits intimes trahissent à quel point il le faisait pour fortifier ou conserver ses positions au sein de la hiérarchie du parti et combien il s'engageait dans des petites tactiques de cliques où ses adversaires 
n'étaient pas les seuls à compter sur le soutien de leurs troupes fidèles et de patrons puissants. Certes, il était persuadé d'intriguer dans les intérêts du parti. Ses actions furent malgré tout fort éloignées des préceptes moraux qu'il prêchait et des principes qu'il prenait pour siens. Jaroslavskij s'est repenti après qu'il a été mis en cause pour de prétendues erreurs idéologiques mais il ne s'inquiétait pas moins des conséquences de l'affaire pour son statut au sein de l'élite que de la pureté des théories consacrées.

L'abîme qui sépare certitudes et pratiques est particulièrement évident dans les parties où Dahlke a rassemblé et analysé une documentation unique sur le quotidien, les vues et les stratégies d'adaptation d'un bolchevik somme toute typique du milieu des dignitaires qui furent conditionnés par le culte qu'ils vouaient à Stalin. L'admiration et le dévouement furent sincères. L'interprétation de Dahlke donne raison à Barbara Walker pour qui la socialisation de l'intelligentsia russe autour de fortes figures d'autorité fournit un modèle pour comprendre les bolcheviks dont la formation et le devenir furent étroitement liés à leur appartenance à des cercles d'étude et groupes d'action dominés par des leaders révérés et à leur rassemblement autour de l'idole qui a réussi à construire une organisation à partir de troupes passablement hétérogènes : Lenin. L'État-parti était à peine moins disparate que le mouvement pré-révolutionnaire des bolcheviks, son contrôle sur le territoire théoriquement conquis était lacunaire et sa maîtrise des rapports sociaux était incertaine. Peu importe ce qu'on sait aujourd'hui des qualités intellectuelles, politiques et humaines de Stalin. À la limite, peu importe ce qu'en soupçonnaient les contemporains. Une fois devenu le Lenin de son époque, Stalin est devenu incontournable, pour le meilleur et pour le pire.

15 Les analyses de Sandra Dahlke pourraient conduire le lecteur à penser que la situation où se trouvait le pays, les moyens à la disposition du régime, les talents problématiques des officiels et leurs manières de s'acquitter de leurs tâches équivalaient à des contraintes formidables qu'on pouvait expliquer, tant bien que mal, par les exercices idéologiques. Mais si les acrobaties théoriques étaient à même de justifier des actions, elles furent d'une efficacité douteuse pour donner des réponses appropriées aux défis qu'elles occultaient. Les dons de l'homme providentiel n'étaient pas nécessairement plus riches que ceux des cadres. De même, sa vision des contraintes structurelles et ses recettes pour les affronter n'étaient pas nécessairement plus pertinentes que celles de ses subordonnés. Mais sa position de chef irrécusable et les facultés extraordinaires qu'on lui prêtait l'ont rendu un gourou incontestable et ont créé un univers où, sans s'en apercevoir, les individus qui s'en remettaient à son génie se trouvaient soulagés de la responsabilité de chercher à réinventer leur monde.

Or, Sandra Dahlke formule le problème différemment. Elle pense avec Irina Paperno que le dictateur apparaissait comme l'incarnation de l'esprit hégélien de la nécessité historique pour ceux qui investissaient leur foi en lui, la Weltseele que le philosophe croyait voir chevaucher dans les rues de Iéna quand Napoléon traversa la ville en 1806. L'interprétation du lecteur et celle de Dahlke sont complémentaires. Elles conduisent à discerner une position de dépendance qui ne procède pas que de l'assujettissement. Comme la servitude volontaire, chère à La Boétie, elle est aussi fonction des calculs de ceux qui se mettent à la disposition d'un pouvoir tyrannique. Les individus n'en sont pas nécessairement conscients.

17 Loin de là. Rien ne fut plus loin de Jaroslavskij que de s'imaginer soumis à Stalin. Mais Dahlke rend évidente sa volonté d'être proche du prince et d'exhiber sa proximité comme symbole de statut. Cette volonté est présente dans son angoisse quand il perd sa 
place à la rédaction de la Pravda et quand il doit faire son autocritique, dans la compétition caricaturale où il s'engage avec d'autres auteurs (dont Mihail Bulgakov) pour écrire une biographie autorisée de Stalin, dans sa joie de se voir reconduit comme membre de l'organe de contrôle du Comité central et dans la satisfaction que lui procure l'honneur d'être choisi pour participer à la rédaction du fameux Précis de l'histoire du PCUS.

Adorno, Horkheimer et Marcuse seraient heureux de découvrir que, même si Dahlke n'utilise pas la catégorie exactement comme eux, un chercheur ose s'aventurer sur leur terrain en mettant dans la perspective de l'aliénation un sujet qui se comprend souverain. Le concept de l'aliénation est quelque peu négligé sans doute en raison de l'erreur fréquente de le prendre uniquement comme l'héritage d'un marxisme vulgaire. L'historienne en fait excellent usage pour clarifier les conséquences de l'identification de Jaroslavskij à la figure de Stalin et à sa politique alors qu'il se perçoit fidèle au projet émancipateur qui le motivait aux débuts de son itinéraire révolutionnaire. Elle relève, par exemple, qu'au fur et à la mesure que le vieux bolchevik s'inféode au leader suprême et à la ligne sinueuse du parti, il se décrit de moins en moins dans son journal intime comme sujet d'actions mais de plus en plus comme objet de circonstances qu'il ne saisit guère, malgré sa conviction du contraire. Qu'il écrive des œuvres théoriques ou historiques, des lettres personnelles ou des notes dans son journal, Jaroslavskij s'exerce à se plier aux règles du jeu d'un univers qui le dépasse. Sans qu'il puisse s'en rendre compte, il se discipline et il se force à s'adapter même quand il s'attèle à des travaux sur le bolchevisme ou sur le passé qu'il fabrique en série non seulement pour légitimer le régime mais aussi afin de se prouver à lui-même la justesse de ses choix.

L'une des grandes frustrations de Jaroslavskij fut que Stalin s'était éloigné de lui après leur lutte commune des années 1920. Le Guide avait besoin de personnages plus rompus à la gestion quotidienne des affaires et aux intrigues politiques de nouveaux types que de l'agitateur ardent. L'ancien combattant ne réussit jamais à regagner la proximité du dictateur. Il était à la lettre de la mort quand sa femme demanda à Stalin de soulager par un geste ses souffrances ainsi que sa douleur de se sentir isolé de la haute direction. Le Secrétaire général essaya de téléphoner mais il ne put parler avec le malheureux. Malgré tout, après les funérailles, l'épouse informa le nouveau Lenin que son admirateur pleurait de bonheur quand elle lui avait fait part des vœux du grand homme.

20 Ce ne fut sans doute pas la fin d'un révolutionnaire indomptable que Jaroslavskij s'imaginait être. Mais c'était sans doute une fin digne de la carrière d'un fidèle militant de la cause bolchevique.

21 Sandra Dahlke a écrit un ouvrage qui fait date. C'est la première fois que le lecteur peut suivre dans le concret les heurs et malheurs de l'existence et de la vie intérieure d'un bâtisseur du système soviétique qui voulait renverser l'Ancien Régime pour faire régner la liberté et finit captif du monde qu'il avait construit, malgré les honneurs dont il fut comblé mais sans qu'il eût à partager le destin des millions de prisonniers qui finirent derrière les barbelés ou devant le peloton d'exécution. Nombre de collègues du propagandiste exalté ont connu le sort des derniers après avoir rempli d'autres fonctions que lui au sein de l'État-parti. Mais le paradigme que Dahlke a réussi à dégager est valable pour la plupart des pairs de Jaroslavskij. Qui sait, il s'applique peut-être à quelques vieux radicaux qui s'agitent sous d'autres cieux et se convainquent à tout prix que le régime qu'ils servent est celui de leurs rêves d'antan ou même mieux. 